\title{
Lambl's excrescences: A case report and review of the literature
}

\author{
Haroon Kamran ${ }^{1}$, Nirav Patel ${ }^{1}$, Gagandeep Singh ${ }^{1}$, Venu Pasricha ${ }^{2}$, Moro Salifu ${ }^{2}$ and Samy I. McFarlane ${ }^{2}$ \\ ${ }^{1}$ Division of Cardiovascular Medicine, State University of New York Downstate Medical Center, Brooklyn, New York, USA \\ ${ }^{2}$ Department of Internal Medicine, State University of New York Downstate Medical Center, Brooklyn, New York, USA
}

\begin{abstract}
Lambl's excrescences are filamentous extensions of cardiac valves, mostly asymptomatic but rarely associated with catastrophic thromboembolic events such as acute ischemic stroke and acute coronary syndromes. Numerous case reports cited in the literature have addressed various spectrum of presentation of these syndromes and their outcomes based on therapies used. We encountered such a patient from our own experience who presented with an acute ischemic stroke with no other identifiable cause other than the Lambl's excrescences. We subsequently carried out an extensive literature search and based on our interpretation of the outcomes we decided to treat the patient to the best of our understanding. In the proceeding section we describe the case and the discussion and our rationale to treat the patient accordingly.
\end{abstract}

\section{Introduction}

Cardioembolism accounts for nearly $20 \%$ of all ischemic strokes [1]. Commonly, it occurs due to embolism of thrombotic material that detaches from atrial, ventricular, or left-sided heart valves. Although not common, Lambl's excrescences have been identified as a cause of cardioembolic stroke [2].

LE are rare cardiac structures described as fine, mobile, filiform fronds that typically occur at sites of valve closure, and are believed to result from minor endothelial damage due to valve wear and tear [3-5]. The differential diagnosis for these excrescences includes fibroelastoma, myxoma, thrombi, vegetations, cardiac neoplasms, and metastases [6]. First described in 1856 by Bohemian physician Vilém Dušan Lambl [7] many clinicians consider LE to be part of the normal aging process. Since its description, several case reports about LE have been published in the medical literature. The clinical significance of LE remains controversial $[8,9]$ and the effective treatment of LE has not been established. Herein, we report a recent case of LE and provide a review of other case reports in the literature.

\section{Case report}

We present a case of Lambl's excrescences associated with ischemic stroke that could not be explained by any other embolic source. The patient is a 74-year-old woman with hypertension, diabetes mellitus, and osteoarthritis who initially presented to the emergency department (ED) with frontal headaches associated with rhinorrhea. The initial impression in the ED was of sinusitis and she was discharged home on nasal decongestants and non-steroidal anti-inflammatory (NSAID). Several hours later, her family noticed sudden weakness in her left upper and lower extremities and brought her back. On arrival in the $\mathrm{ED}$, stroke code was initiated and the patient was evaluated by the neurology service. The initial evaluation revealed markedly decreased left-sided upper and lower motor function with intact pain, vibration, temperature and proprioception. A stat non-contrast computerized tomography (CT) scan revealed an acute hypodensity in the right lentiform nucleus territory, consistent with acute ischemic stroke (Figure 1A). Her National Institute of Health Stroke Scale (NIHSS) score was found to be five and deemed outside the thrombolytic window. She was admitted to the neurology service for further management of ischemic stroke and evaluation of the embolic source.

Over the course of her admission, she was started on anti-platelet therapy with aspirin (ASA), high-intensity statin, IV hydration, and permissive hypertension with a goal blood pressure of $>220 / 110 \mathrm{mmHg}$ as per the current neurovascular guidelines. As part of the workup, MRI of the head, MRA of the neck, carotid dopplers, and transthoracic echocardiograms (2D Echo) were ordered to determine the probable source of her ischemic stroke. She was also placed on continuous

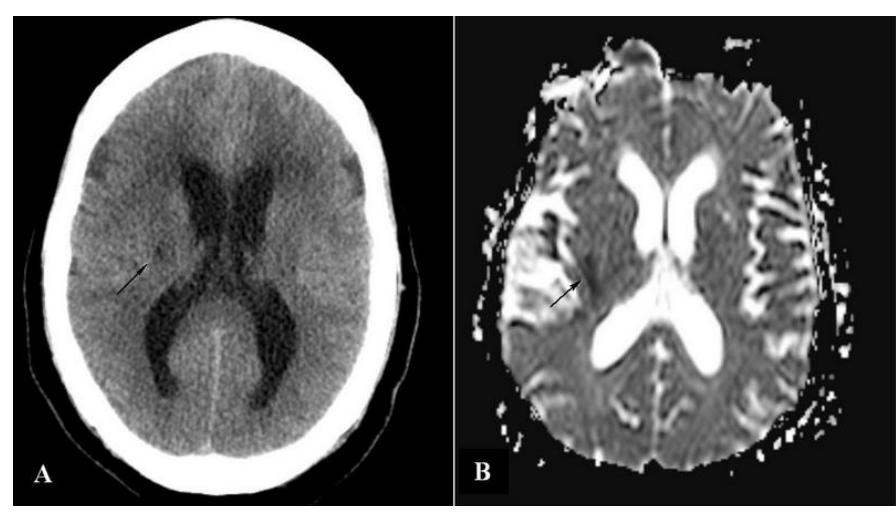

Figure 1. Arrows showing ischemic stroke in the lentiform nucleus territory on CT scan (A) and MRI (B).

Correspondence to: Samy I. McFarlane, MD, MPH, MBA, FACP, Professor of Medicine and Endocrinology, State University of New York-Downstate Medical Center, 450 Clarkson Avenue, Box 50 Brooklyn, New York, 11203, Tel: 718-2703711; Fax: 718-270-6358; E-mail: Samy.McFarlane@downstate.edu

Received: June 29, 2016; Accepted: July 20, 2016; Published: July 25, 2016 
telemetry monitoring to evaluate for possible arrhythmias. Further lab work included lipid panel and hemoglobin A1C measurement for cardiovascular risk stratification.

Over the course of her hospital stay, her motor function did not improve significantly. The 12-lead electrocardiogram (ECG) on admission and on serial monitoring demonstrated normal sinus rhythm. The telemetry monitor did not show any arrhythmias. The MRI of the head confirmed the presence of the ischemic stroke in the lentiform nucleus territory without any obvious surrounding edema or midline shift (Figure 1B). The MRA of the neck and the carotid doppler did not reveal any significant stenosis or atherosclerosis. The 2D showed preserved left ventricular (LV) systolic function with impaired LV relaxation consistent with grade 1 diastolic dysfunction. Given the paucity of evidence concerning the source of the embolic stroke, a trans-esophageal echocardiogram (TEE) was ordered for further evaluation. The TEE showed no left atrial appendage thrombus or spontaneous echo contrast but was notable for a small echo density on the anterior aortic valve leaflets, consistent with Lambl's excrescences (Figure 2).

After the discovery of the Lambl's excrescences, a detailed review of the literature was carried out to see if there were any guidelines for the optimal management of ischemic stroke in such patients. We did not find any consensus on medical or surgical therapy for patients with Lambl's excrescences. Different authorities have used different approaches, which have ranged from single anti-platelet therapy to full-dose anticoagulation to surgical intervention. A multi-disciplinary meeting was held to discuss the patient, which included specialists from general cardiology, non-invasive cardiology, and internal medicine. The consensus was that we should start the patient on life-long, fulldose anti-coagulation therapy for secondary prevention of further ischemic strokes. This strategy was adopted after a careful review and discussion with the patient and her family regarding the risks and benefits of therapy.

Based on extensive review of the literature, in the following section we discuss the different situations and therapy regimens with possible follow-ups where applicable for the management of Lambl's excrescences.

\section{Discussion}

Lambl's excrescences are asymptomatic and occur most commonly on the mitral (atrial surface) and aortic (ventricular surface) valves. They have been rarely described on native pulmonary, tricuspid valves and prosthetic valves. A conglomeration of multiple Lambl's excrescences may detach from the cardiac valve and lead to peripheral embolization. Evidence suggests an association between Lambl's excrescences and embolic events and acute coronary syndromes [4,10,11]. There have been case reports on giant LEs ( $\geq 2 \mathrm{~cm}$ in diameter) associated with ischemic stroke [11-14]. However, no clear evidence is found showing the correlation between the strand size and potential risk of
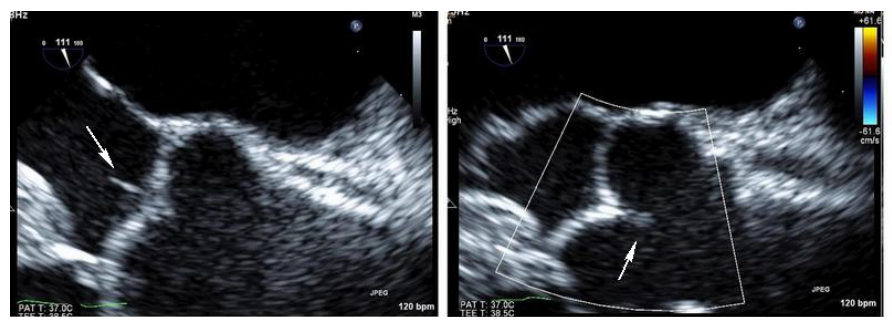

Figure 2. Transesophageal echo showing Lambl'sexcresences (Arrows). thromboembolic event. Ischemic events are more commonly observed in LE of the aortic valve.

An evidence-based approach to the management and treatment of LE has yet to be established. Chu et al suggest that physicians should consider LE in the differential diagnosis for a patient with cryptogenic stroke [15]. They recommend performing a trans-esophageal echocardiography (TEE) in this population, and if LE is present, treatment with dual anti-platelet therapy should be considered. Furthermore, if a recurrent ischemic event occurs while on this therapy, a trial of anticoagulation therapy should be considered before proceeding with surgical resection of the LE. Most authors agree that the choice between surgical intervention versus conservative observation, including anticoagulation, needs to be decided on a case-by-case basis and should be based on the pre-operative risk stratification for the patient [11].

Because there is no consensus on the treatment of LE, the management is largely based upon case reports in the literature. The general therapeutic approach to LE with a single embolic ischemic event is either anticoagulation or anti-platelet therapy. Kalavukunta et al. [4] presented a 59-year-old male with LE and acute infarction of both cerebral hemispheres with embolic phenomena who was subsequently started on anticoagulation therapy with warfarin. Aggrawal et al. [13] reported a case of a 66-year-old female with large LE on her aortic valve with infarction of the parietal subcortical region. Consequently, the patient was started on anticoagulation therapy but 3 weeks later she had another stroke even though she was receiving full dose anticoagulation therapy, leading to surgical intervention. Wu et al. [12] presented the case of a 66-year-old female with embolic infarction of the left posterior cerebral artery who was treated with warfarin; during 6 months of follow-up, the patient did not have any neurologic events.

Antiplatelet agents, such as aspirin, has been recognized as potential therapy following a single embolic event (Davogustto et al. [11], Wolf et al. [16], Siles et al. [17], Liu et al. [18]). Chu et al. [15] also reported a case of a 51-year-old female with two ischemic strokes within 3 months found to have LE on her aortic valve and started on aspirin and Clopidogrel.

As noted, there is no consensus in the literature about whether to use anticoagulation versus anti-platelet therapy in a patient with a single embolic event due to LE. However, based on these case reports and the literature review, it seemed prudent to start warfarin in our patient. We recommended that the patient have close follow-up and surveillance for another neurological event.

\section{Conclusion}

A review of the literature shows several different views on the clinical significance of LE and their relationship to ischemic events. These different opinions are based mostly on scant evidence. Although they are rare and their clinical significance is debated, LE should be considered in patients who present with signs of cardioembolism. The optimal treatment for patients with LE remains to be determined.

\section{Acknowledgement}

This work is sponsored in part by the Brooklyn Health Disparities Center NIH grant \#P20 MD006875.

\section{References}

1. Murtagh B, Smalling RW (2006) Cardioembolic stroke. Curr Atheroscler Rep 8: 310316. [Crossref] 
2. Leary MC, Caplan LR: Cardioembolic stroke: An update on etiology, diagnosis and management. Ann Indian Acad Neurol 11: 52-55.

3. Jaffe W, Figueredo VM (2007) An example of Lambl's excrescences by transesophageal echocardiogram: a commonly misinterpreted lesion. Echocardiography 24: 1086-1089. [Crossref]

4. Kalavakunta JK, Peddi P, Bantu V, Tokala H, Kodenchery M (2010) Lambl's excrescences: a rare cause of stroke. J Heart Valve Dis 19: 669-670. [Crossref]

5. Al-Ansari S, Hindori V, Riezebos RK, Yilmaz A (2013) Multiple Lambl's excrescences with subvalvular extension, a rare cause of cryptogenic stroke: treated by port-access cardiac surgery. BMJ Case Rep 2013. [Crossref]

6. Morgan JA, Paone G (2012) Resection of Lambl's excrescence on the aortic valve in a patient with rheumatic mitral valve disease and a left atrial thrombus. Heart Surg Forum 15: E215-217. [Crossref]

7. Lambl VD (1856) Papillare exkreszenzen an der semilunar-klappe der aorta. Wien Med Wochenschr 6: 244-7.

8. Roldan CA, Shively BK, Crawford MH (1997) Valve excrescences: prevalence, evolution and risk for cardioembolism. J Am Coll Cardiol 30: 1308-1314. [Crossref]

9. Freedberg RS, Goodkin GM, Perez JL, Tunick PA, Kronzon I (1995) Valve strands are strongly associated with systemic embolization: a transesophageal echocardiographic study. J Am Coll Cardiol 26: 1709-12.

10. Cohen A, Tzourio C, Chauvel C, Bertrand B, Crassard I, et al. (1995) Mitral valve fibrin strands detected by transesophageal echocardiography are a risk factor for ischemic stroke: a case control study in patients aged over 60 [abstract]. Circulation 92: I-285.

11. Davogustto G, Fernando RR, Loghin C (2015) Lambl's excrescence, migrainous headaches, and "tiger stripes": puzzling findings in one patient. Tex Heart Inst $J 42$ : 70-72. [Crossref]

12. Wu TY, Gerber IL, Roxburgh RH (2013) Thrombo-embolic cerebral infarction secondary to giant Lambl's excrescence. J Clin Neurosci 20: 1632-1634. [Crossref]

13. Aggarwal A, Leavitt BJ (2003) Images in clinical medicine. Giant Lambl's excrescences. $N$ Engl J Med 349: e24. [Crossref]

14. Homma S, Di Tullio MR, Sciacca RR, Sacco RL, Mohr JP; PICSS Investigators (2004) Effect of aspirin and warfarin therapy in stroke patients with valvular strands. Stroke 35: 1436-1442. [Crossref]

15. Chu A, Aung TT, Sahalon H, Choksi V, Feiz H (2015) Lambl's Excrescence Associated with Cryptogenic Stroke: A Case Report and Literature Review. Am J Case Rep 16 876-881. [Crossref]

16. Wolf RC, Spiess J, Huber R (2006) [Lambl's excrescence and cerebral ischemic insult] Nervenarzt 77: 1492-1494. [Crossref]

17. Siles Rubio JR, Ruiz de Castroviejo del Campo J, Tirado Miranda R, Jansen Chaparro S, Pavlovic D (2006) [Transient ischemic attack due to Lambl's excrescence. Report of a case and review of the literature]. An Med Interna 23: 181-183. [Crossref]

18. Liu RZ, Yu SY, Li Y (2012) Migraine-like headache and ischemic strokes in two patients with Lambl's excrescences. Chin Med J (Engl) 125: 3346-3348. [Crossref]

Copyright: (92016 Kamran H. This is an open-access article distributed under the terms of the Creative Commons Attribution License, which permits unrestricted use, distribution, and reproduction in any medium, provided the original author and source are credited. 